Article

\title{
Data-Driven Model-Free Adaptive Control Based on Error Minimized Regularized Online Sequential Extreme Learning Machine
}

\author{
Xiaofei Zhang ${ }^{1}$ and Hongbin Ma ${ }^{2, *}$ \\ 1 School of Automation, Beijing Institute of Technology, Beijing 100081, China \\ 2 State Key Laboratory of Intelligent Control and Decision of Complex Systems, \\ Beijing Institute of Technology, Beijing 100081, China \\ * Correspondence: mathmhb@bit.edu.cn or 3120170444@bit.edu.cn; Tel.: +86-10-68913985
}

Received: 18 July 2019; Accepted: 7 August 2019; Published: 22 August 2019

check for updates

\begin{abstract}
Model-free adaptive control (MFAC) builds a virtual equivalent dynamic linearized model by using a dynamic linearization technique. The virtual equivalent dynamic linearized model contains some time-varying parameters, time-varying parameters usually include high nonlinearity implicitly, and the performance will degrade if the nonlinearity of these time-varying parameters is high. In this paper, first, a novel learning algorithm named error minimized regularized online sequential extreme learning machine (EMREOS-ELM) is investigated. Second, EMREOS-ELM is used to estimate those time-varying parameters, a model-free adaptive control method based on EMREOS-ELM is introduced for single-input single-output unknown discrete-time nonlinear systems, and the stability of the proposed algorithm is guaranteed by theoretical analysis. Finally, the proposed algorithm is compared with five other control algorithms for an unknown discrete-time nonlinear system, and simulation results show that the proposed algorithm can improve the performance of control systems.
\end{abstract}

Keywords: model free adaptive control; machine learning; data-driven control

\section{Introduction}

It is difficult to obtain an accurate mechanism model of a physical system when the production technologies and processes are very complex. Data-driven control (DDC) [1] relies on input/output (I/O) data of control systems and does not need to consider mechanism models of systems. After several years of development, some data-driven control techniques have been investigated, such as, proportional-integral derivative (PID) [2], fuzzy logic control [3], unfalsified control (UC) [4,5], model free adaptive control (MFAC) [6-12], iterative learning control (ILC) [13-15], iterative feedback tuning (IFT) $[16,17]$, some control algorithms based on neural network [18-23] and so on.

MFAC is a class of DDC, and it builds a virtual equivalent dynamic linearized model [24] by using a dynamic linearization technique. The virtual equivalent dynamic linearized model contains some time-varying parameters. In practice, it is often not easy to obtain the resulting time-varying parameters, but they can be estimated by using historical data of control systems. Those time-varying parameters usually include high nonlinearity implicitly, and the performance will degrade if the nonlinearity of the resulting time-varying parameters is too severe [25]. The traditional methods of getting those time-varying parameters are projection algorithm, recursive least squares, and so on. Besides every time-varying parameter in the virtual equivalent dynamic linearized model can be considered as a nonlinear function, and in [25], radial basis function neural network (RBFNN) is used to estimate those time-varying parameters. 
Extreme learning machine (ELM) is developed for offline learning and training a single-hiddenlayer feed forward neural networks (SLFNs) [26]. Online sequential extreme learning machine (OS-ELM) [27] is an online learning algorithm, it adjusts the output weights online, besides the input weights and hidden biases are randomly chose. In recent years OS-ELM has gained a large amount of interests and been uesed to estimate unknown parameters of systems [28,29]. Some improved OS-ELM algorithms were introduced by some scholars, such as regularized online sequential extreme learning machine (REOS-ELM) [30], initial-training-free online extreme learning machine (ITF-OELM) [31], etc. Although, the learning speed of OS-ELM is extremely high, it could yield singular and ill-posed problens. To overcome adverse effects, which cuased by the noisy data of control systems, REOS-ELM, which is an improvement of OS-ELM, was investigated. In [30], REOS-ELM was introduced, however the stability of REOS-ELM for unknown discrete-time nonlinear systems was not analysed, and how to select the hidden node number of neural networks is unknown.

In this paper, first, a updating formula which contains dead-zone characteristics for REOS-ELM is introduced. Second, in order to obtain a more compact network structure, error minimized regularized online sequential extreme learning machine (EMREOS-ELM) is investigated, EMREOS-ELM is different from EMOS-ELM [28], and it updates the output weights using the pseudoinverse of a partitioned matrix during the growth of the networks. Finally, a novel model-free adaptive control method based on EMREOS-ELM is proposed. This paper is structured as follows, in Section 2 REOS-ELM is briefly introduced. Dynamic linearization technique and the updating formula which contains dead-zone characteristics for REOS-ELM are introduced in Section 3. The model-free adaptive control method based on EMREOS-ELM and the stability analysis of the proposed method for unknown discrete-time nonlinear systems are stated in Section 4. Section 5 shows simulation experiments. In Section 6 some conclusions are brought.

\section{REOS-ELM}

In [32], the universal approximation capability of ELM was analyzed. ELM is developed for offline learning, however training data could arrive one-by-one or chunk-by-chunk; OS-ELM is an online version of ELM, and it is sensitive to noise data; REOS-ELM is an improvement of OS-ELM. In this part REOS-ELM will be introduced.

Suppose that there is an initial training dataset with $N_{0}$ training patterns $\left(x_{j}, t_{j}\right) \in \boldsymbol{R}^{n} \times \boldsymbol{R}$ where $x_{j}=\left[x_{j 1}, x_{j 1}, \cdots, x_{j n}\right]^{T}$. For a single hidden layer neural network with $n$ input nodes and one output node, the $j$ th output corresponding to the $j$ th input pattern is

$$
o_{j}=\sum_{i=1}^{L} \beta_{i} G\left(\boldsymbol{w}_{i}, b_{i}, \boldsymbol{x}_{j}\right), j=1, \cdots, N_{0}
$$

where $L$ denotes the number of hidden nodes, and the subscript $i$ indicates the $i$ th hidden node; $G(\cdot)$ and $o_{j}$ are the active function of hidden nodes and the output of the network, respectively, $b_{i}$ and $\beta_{i}$ are the hidden bias of the $i$ th hidden node and the output weight connecting the $i$ th hidden layer and the output node, respectively.

OS-ELM is a novel online learning algorithm due to its highlighted features, and its highlighted features are that the input weights and hidden biases are randomly chose, and the main goal of training process is to determine the output weights. In order to obtain output weights, they minimize the error function defined by

$$
\left\|H_{0} \beta_{0}-T_{0}\right\|^{2} .
$$

The collected data for training process often contains noise, and the only objective of empirical risk minimization as shown in (2) may lead to poor generalization and over-fitting [30]. REOS-ELM is 
different from OS-ELM, and REOS-ELM tries to minimize the empirical error and obtain small norm of network weight vector [30]. In [30] the followin cost function was considered, and the cost function is

$$
\left\|H_{0} \beta_{0}-T_{0}\right\|^{2}+\lambda\left\|\beta_{0}\right\|^{2}
$$

where $\lambda$ is a regularization factor.

The solution of $\beta_{0}$ is

$$
\beta_{0}=P_{0} H_{0}^{T} T_{0}
$$

and

$$
\boldsymbol{P}_{0}=\left(\boldsymbol{H}_{0}^{T} \boldsymbol{H}_{0}+\lambda \boldsymbol{I}_{L}\right)^{-1}
$$

where $\boldsymbol{I}_{L}$ is an identity matrix, the size of $\boldsymbol{I}_{L}$ is $L, \boldsymbol{T}_{0}=\left[t_{1}, \cdots, t_{N_{0}}\right]^{T}$, and

$$
\boldsymbol{H}_{0}=\left[\begin{array}{ccc}
G\left(\boldsymbol{w}_{1}, b_{1}, \boldsymbol{x}_{1}\right) & \cdots & G\left(\boldsymbol{w}_{L}, b_{L}, \boldsymbol{x}_{1}\right) \\
\vdots & \cdots & \vdots \\
G\left(\boldsymbol{w}_{1}, b_{1}, \boldsymbol{x}_{N_{0}}\right) & \cdots & G\left(\boldsymbol{w}_{L}, b_{L}, \boldsymbol{x}_{N_{0}}\right)
\end{array}\right]_{N_{0} \times L}
$$

When the $k$ th chunk of data is recerived

$$
N_{k}=\left\{\left(\boldsymbol{x}_{i}, t_{i}\right)\right\}_{i=\sum_{j=0}^{k-1} N_{j}+1}^{i=0} N_{j}
$$

The weight updating algorithm, which is used in REOS-ELM, takes a similar form to recursive least squares (RLS) algorithm, and

$$
\boldsymbol{P}_{k}=\boldsymbol{P}_{k-1}-\frac{\boldsymbol{P}_{k-1} \boldsymbol{H}_{k}^{T} \boldsymbol{H}_{k} \boldsymbol{P}_{k-1}}{\boldsymbol{I}_{N_{k}}+\boldsymbol{H}_{k} \boldsymbol{P}_{k-1} \boldsymbol{H}_{k}^{T}}
$$

and

$$
\boldsymbol{\beta}_{k}=\boldsymbol{\beta}_{k-1}+\boldsymbol{P}_{k} \boldsymbol{H}_{k}^{T}\left(\boldsymbol{T}_{k}-\boldsymbol{H}_{k} \boldsymbol{\beta}_{k-1}\right)
$$

where $\boldsymbol{I}_{N_{k}}$ is an identity matrix, and the size of $\boldsymbol{I}_{N_{k}}$ is $N_{k} ; \boldsymbol{T}_{k}$ and $\boldsymbol{H}_{k}$ are the target of $k$ th arriving training data and the hidden layer outputs for the $k$ th arriving training data, respectively.

\section{Dynamic Linearization Technique and the New Updating Formula for REOS-ELM}

\subsection{Dynamic Linearization Technique}

MFAC builds a virtual equivalent dynamic linearized model by using a dynamic linearization technique.

The following unknown discrete-time nonlinear system is considered

$$
y_{k+1}=f\left(y_{k}, \cdots, y_{k-L_{y}}, u_{k}, \cdots, u_{k-L_{u}}\right)
$$

where $f(\cdot)$ represents an unknown nonlinear function, and here $L_{y}$ and $L_{u}$ indicate the orders of the system input and the system output, respectively.

To make further study, the following assumptions are used.

Assumption 1. The system (10) is observable and controllable.

Assumption 2. The $f(\cdot)$ is a smooth nonlinear function, and the partial derivative of $f(\cdot)$ with respect to $u_{k}$ is continuous. 
Assumption 3. Suppose that for all $k \in 0,1, \cdots, T$, and if $\triangle u_{k} \neq 0$, then system (10) satisfies the generalized Lipschitz condition along the iteration axis, that is

$$
\left|\triangle y_{k+1}\right| \leq L_{b}\left|\triangle u_{k}\right|
$$

where $\triangle y_{k+1}=y_{k+1}-y_{k}, \Delta u_{k}=u_{k}-u_{k-1}, L_{b}$ is a constant, and $L_{b}>0$.

Remark 1. This assumption means that bounded change of the input can not cause unbounded change of the system output, which can be guaranteed by many industrial processes [25].

Theorem 1. For the unknown discrete-time nonlinear system (10), satisfying Assumptions (Remarks 1-3), there must be $\psi_{k}$ called pseudo-partial-derivative (PPD), when $\triangle u_{k} \neq 0$, the system (10) can be rewritten as

$$
\triangle y_{k+1}=\psi_{k} \triangle u_{k}
$$

where $\left|\psi_{k}\right| \leq L_{b}$.

Proof. See ([33] theorem 4.1).

Assumption 4. For the unknown discrete-time nonlinear system (10), satisfying Assumptions (Remarks 1-3), there is $\boldsymbol{\beta}^{*}$, when $\triangle u_{k} \neq 0$, the system (10) can be rewritten as

$$
y_{k+1}=y_{k}+\boldsymbol{H}_{k} \boldsymbol{\beta}^{*} \triangle u_{k}+\triangle_{k} \triangle u_{k}
$$

where

$$
\boldsymbol{H}_{k} \boldsymbol{\beta}^{*}=\sum_{i=1}^{L} \beta_{i}^{*} G\left(\boldsymbol{w}_{i}, b_{i}, \boldsymbol{x}_{k}\right)
$$

$\sup \left|\triangle_{k}\right| \leq \triangle$, and $\triangle$ is a given upper bound; $w_{i}, b_{i}$ are randomly generated, then they kept in constant.

3.2. The Updating Formula Based on Dead-Zone Characteristics

The discrete time nonlinear system (10) is a class of special system where the training data comes one by one, and $N_{k}=1$.

When $\boldsymbol{H}_{k} \hat{\boldsymbol{\beta}}_{k} \neq 0$, and $\left|\frac{y_{k+1}^{*}-y_{k}}{\boldsymbol{H}_{k} \hat{\beta}_{k}}\right| \leq M$, the control law is

$$
u_{k}=\frac{y_{k+1}^{*}-y_{k}}{\boldsymbol{H}_{k} \hat{\boldsymbol{\beta}}_{k}}+u_{k-1}
$$

where $M$ is a constant, $M>0, y_{k+1}^{*}$ is the desired signal and $\hat{\boldsymbol{\beta}}_{k}$ is defined in below.

When $\boldsymbol{H}_{k} \hat{\boldsymbol{\beta}}_{k}=0$, or $\left|\frac{y_{k+1}^{*}-y_{k}}{\boldsymbol{H}_{k} \hat{\boldsymbol{\beta}}_{k}}\right|>M$, or $\Delta \boldsymbol{u}_{k}=0$, a new re-learning process begins.

The updating formula, which contains dead-zone characteristics, for REOS-ELM is

$$
\boldsymbol{P}_{k}^{-1}=\boldsymbol{P}_{k-1}^{-1}+\boldsymbol{H}_{k}^{T} \boldsymbol{H}_{k} \sigma_{k}
$$

and

$$
\hat{\boldsymbol{\beta}}_{k+1}=\hat{\boldsymbol{\beta}}_{k}+\sigma_{k} \boldsymbol{P}_{k} \boldsymbol{H}_{k}^{T} e_{k+1}^{*}
$$

where

$$
\sigma_{k}=\left\{\begin{array}{cc}
1 & \left(\frac{\left|e_{k+1}^{*}\right|}{1+\sigma_{k} \boldsymbol{H}_{k} \boldsymbol{P}_{k-1} \boldsymbol{H}_{k}^{T}}\right)^{2}>\triangle^{2} \\
0 & \text { otherwise }
\end{array}\right.
$$


and

$$
e_{k+1}^{*}=\frac{y_{k+1}-y_{k+1}^{*}}{\triangle u_{k}}
$$

Lemma 1. If

$$
\boldsymbol{P}_{k}^{-1}=\boldsymbol{P}_{k-1}^{-1}+\boldsymbol{H}_{k}^{T} \boldsymbol{H}_{k} \sigma_{k}
$$

where the scalar $\sigma_{k}>0$, then $\boldsymbol{P}_{k}$ is related to $\boldsymbol{P}_{k-1}$ via

$$
\boldsymbol{P}_{k}=\boldsymbol{P}_{k-1}-\frac{\sigma_{k} \boldsymbol{P}_{k-1} \boldsymbol{H}_{k}^{T} \boldsymbol{H}_{k} \boldsymbol{P}_{k-1}}{1+\sigma_{k} \boldsymbol{H}_{k} \boldsymbol{P}_{k-1} \boldsymbol{H}_{k}^{T}}
$$

and

$$
\boldsymbol{P}_{k} \boldsymbol{H}_{k}^{T}=\frac{\boldsymbol{P}_{k-1} \boldsymbol{H}_{k}^{T}}{1+\sigma_{k} \boldsymbol{H}_{k} \boldsymbol{P}_{k-1} \boldsymbol{H}_{k}^{T}} .
$$

Proof. It is a direct conclusion of [33].

\section{The MFAC Method Based on EMREOS-ELM}

In this section, we will introduce the MFAC method based on EMREOS-ELM, and the MFAC method based on EMREOS-ELM is performed in three main phases, and they are parameter initialization, parameter learning and the adjustment of network structure. EMREOS-ELM is an online learning algorithm and it can train a single hidden layer neural network, besides it can adjust the network structure; When the system is initialized or the network structure is adjusted, EMREOS-ELM need much training data, which is used for the training network structure. In this paper, the size of those training data is 200, than is, $K_{m}=200 I_{m}=200$; The maximum number of hidden nodes and the minimum number of hidden nodes are usually selected based on the complexity of unknown discrete-time nonlinear systems. Figure 1 illustrates the process of the proposed algorithm. 


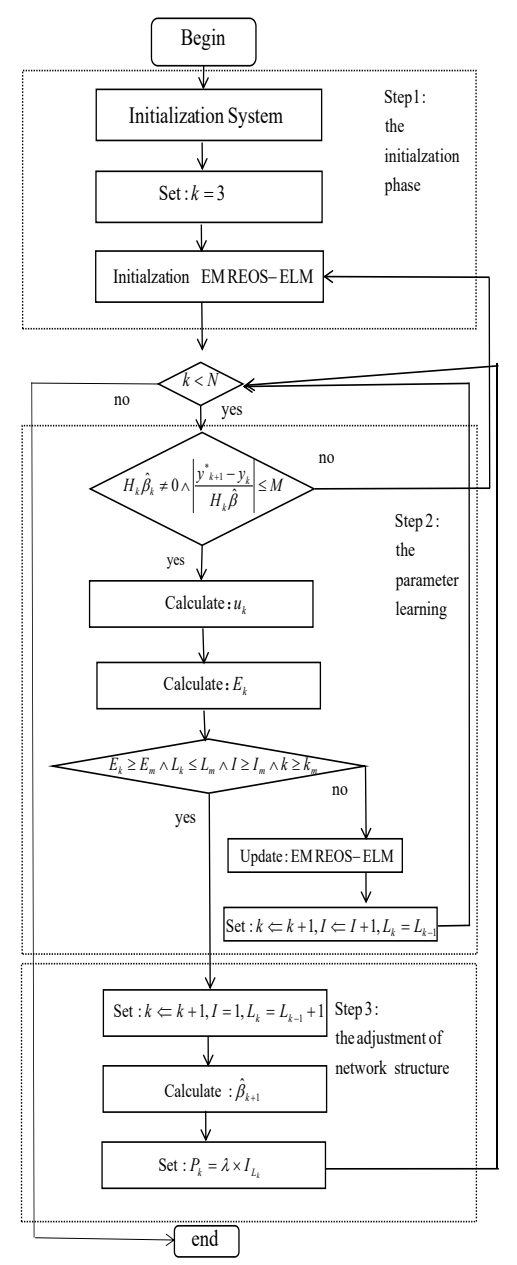

Figure 1. the process of the proposed MFAC method based on EMREOS-ELM.

\subsection{Initialization Phase}

1. $u_{1}$ and $u_{2}$ are two random values, $\left|u_{1}\right| \leq 1\left|u_{2}\right| \leq 1$, and set $k=3$.

2. Set $M=100, L_{m}=20$, and $L_{1}=L_{2}=8$. $L_{m}$ denotes the maximum number of hidden nodes, and $L_{1}$ and $L_{2}$ denote the minimum number of hidden nodes.

3. Measure the output $y_{2}$ and $y_{3}$ of system (10).

4. Assign random parameters of hidden nodes $\left(w_{i}, b_{i}\right)$ where $i=1, \cdots, L_{2}, \lambda=0.1$.

5. Using the first sample data $\boldsymbol{x}_{1}=\left[y_{2}, u_{1}\right]$, initialize $\boldsymbol{P}_{1}$ and $\hat{\boldsymbol{\beta}}_{2}$, and

$$
\begin{gathered}
\boldsymbol{P}_{0}=\left(\lambda \boldsymbol{I}_{L_{2}}\right)^{-1}, \\
\boldsymbol{P}_{1}=\left(\lambda \boldsymbol{I}_{L_{2}}+\boldsymbol{H}_{1}^{T} \boldsymbol{H}_{1}\right)^{-1},
\end{gathered}
$$

and

$$
\hat{\boldsymbol{\beta}}_{1}=\boldsymbol{P}_{1} \boldsymbol{H}_{1}^{T} e_{3}^{*}
$$

Define

$$
\hat{\beta}_{2} \triangleq \hat{\beta}_{1}
$$

\subsection{Parameter Learning}

1. Using the $k$ th sample data $\boldsymbol{x}_{k}=\left[y_{k}, u_{k-1}\right]$, calculate $\boldsymbol{H}_{k}$, and 
when $\boldsymbol{H}_{k} \hat{\boldsymbol{\beta}}_{k} \neq 0$, and $\left|\frac{y_{k+1}^{*}-y_{k}}{\boldsymbol{H}_{k} \hat{\boldsymbol{\beta}}_{k}}\right| \leq M$, then

$$
u_{k}=\frac{y_{k+1}^{*}-y_{k}}{\boldsymbol{H}_{k} \hat{\boldsymbol{\beta}}_{k}}+u_{k-1}
$$

when $\boldsymbol{H}_{k} \hat{\boldsymbol{\beta}}_{k}=0$, or $\left|\frac{y_{k+1}^{*}-y_{k}}{\boldsymbol{H}_{k} \hat{\boldsymbol{\beta}}_{k}}\right|>M$ or $\left|\triangle u_{k}\right|=0$, a new re-learning process begins.

2. Measure the output $y_{k+1}$ of system (10).

3. Set $E_{m}=0.05, E_{v}=50$, and calculate $E_{k}$

$$
E_{k}=\sum_{i=k-E_{V}}^{k-1}\left(y^{*}(i)-y(i)\right)^{2} .
$$

where $E_{V}$ and $E_{m}$ are two values, and they are important for adjusting network structure; The appropriate $E_{V}$ and $E_{m}$ can improve the tracking effect of systems, and too small $E_{V}$ or too big $E_{m}$ make EMREOS-ELM become invalid; We can choose those values $E_{V}$ and $E_{m}$ based on performance requirements of systems.

4. When the tracking error does not meet the requirements of systems, network structure will be adjusted. The core of the proposed EMREOS-ELM is the adjustment of network structure. if $E_{k} \geq E_{m} \wedge L_{k} \leq L_{m} \wedge I \geq I_{m} \wedge k \geq K_{m}$ then execute II; or I.

(I)

Using the $k$ th training data $x_{k}=\left[y_{k}, u_{k-1}\right]$ and $e_{k+1}^{*}$, update the output weights

$$
\boldsymbol{P}_{k}=\boldsymbol{P}_{k-1}-\frac{\sigma_{k} \boldsymbol{P}_{k-1} \boldsymbol{H}_{k}^{T} \boldsymbol{H}_{k} \boldsymbol{P}_{k-1}}{1+\sigma_{k} \boldsymbol{H}_{k} \boldsymbol{P}_{k-1} \boldsymbol{H}_{k}^{T}}
$$

and

$$
\hat{\boldsymbol{\beta}}_{k+1}=\hat{\boldsymbol{\beta}}_{k}+\sigma_{k} \boldsymbol{P}_{k} \boldsymbol{H}_{k}^{T} e_{k+1}^{*}
$$

where

$$
\sigma_{k}=\left\{\begin{array}{cc}
1 & \left(\frac{\left|e_{k+1}^{*}\right|}{1+\sigma_{k} \boldsymbol{H}_{k} \boldsymbol{P}_{k-1} \boldsymbol{H}_{k}^{T}}\right)^{2}>\triangle^{2} \\
0 & \text { otherwise }
\end{array},\right.
$$

and

$$
e_{k+1}^{*}=\frac{y_{k+1}-y_{k+1}^{*}}{\triangle u_{k}} .
$$

5. $\quad k \Leftarrow k+1, L_{k}=L_{k-1}, I \Leftarrow I+1$, and go to Section 4.2.

(II) Go to Section 4.3.

\subsection{The Adjustment of Network Structure}

1. Set $L_{k}=L_{k-1}+1$, and assign random parameters of the $L_{k}$ th hidden node $\left(\boldsymbol{w}_{L_{k}}, b_{L_{k}}\right)$.

2. When network structure is adjusted, it is equivalent to add a new column to $\boldsymbol{H}_{k}$, and

$$
\triangle \delta=G\left(\boldsymbol{w}_{L_{k}}, b_{L_{k}}, x_{k}\right) .
$$

Then

$$
\boldsymbol{H}_{k}^{*}=\left[\boldsymbol{H}_{k} \mid \triangle \delta\right] .
$$


3. The pseudo inverse of the new $\left(\boldsymbol{H}_{k}^{*}\right)^{\dagger}$ is

$$
\left(\boldsymbol{H}_{k}^{*}\right)^{\dagger}=\left[\begin{array}{c}
\left(\boldsymbol{H}_{k}\right)^{+}-\boldsymbol{d b} \\
b
\end{array}\right]
$$

where

$$
\boldsymbol{d}=\left(\boldsymbol{H}_{k}\right)^{+} \triangle \delta
$$

and

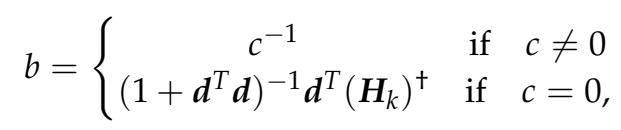

and

$$
c=\triangle \delta-H_{k} d
$$

4. Calculate $\hat{\boldsymbol{\beta}}_{k+1}$, and

$$
\hat{\boldsymbol{\beta}}_{k+1}=\left[\begin{array}{c}
\hat{\boldsymbol{\beta}}_{k}-\boldsymbol{d} b \triangle \delta \\
b \triangle \delta
\end{array}\right]
$$

5. Initialize $\boldsymbol{P}_{k}$ and

$$
\boldsymbol{P}_{k}=\lambda \times \boldsymbol{I}_{L_{k}}
$$

where $\boldsymbol{I}_{L_{k}}$ denotes $L_{k} \times L_{k}$ identify matrix.

6. Set $u_{k}$ as a random number, and $\left|u_{k}\right| \leq 1$.

7. Set $k \Leftarrow k+1, I=1$, and go to Section 4.2.

\subsection{Stability Analysis}

For EMREOS-ELM, the adjustment of network structure and online learning are independent of each other, when the structure of the network is adjusted, online learning is not executed in this period. If the structure, i.e., the hidden node number is settled to a fixed value, EMREOS-ELM will be changed into REOS-ELM. Thus, the stability analysis of the model-free adaptive control method based on EMREOS-ELM can be similarly established the stability analysis of the model-free adaptive control method based on REOS-ELM. In this section, the stability of the model-free adaptive control method based on REOS-ELM is analyzed.

Theorem 2. For the system (10), if the updating formulas described by (16) and (17) are adopted, the following results can be obtained.

(1) $\left\|\boldsymbol{\beta}^{*}-\hat{\boldsymbol{\beta}}_{k}\right\|^{2} \leq \iota_{1}\left\|\boldsymbol{\beta}^{*}-\hat{\boldsymbol{\beta}}_{1}\right\|^{2}$ where $\iota_{1}=\frac{\lambda_{\max }\left(\boldsymbol{P}_{0}^{-1}\right)}{\lambda_{\min }\left(\boldsymbol{P}_{0}^{-1}\right)}$, and $\lambda_{\max }\left(\boldsymbol{P}_{0}^{-1}\right)$ and $\lambda_{\min }\left(\boldsymbol{P}_{0}^{-1}\right)$ are the maximum eigenvalue and the minimum eigenvalue of the matrix $\boldsymbol{P}_{0}^{-1}$, respectively.

(2) $\lim _{N \rightarrow \infty} \sum_{k=1}^{N} \sigma_{k}\left[\left(\frac{\left|e_{k+1}^{*}\right|}{1+\sigma_{k} \boldsymbol{H}_{k} \boldsymbol{P}_{k-1} \boldsymbol{H}_{k}^{T}}\right)^{2}-\triangle^{2}\right]<\infty$

and this implies
(a) $\lim _{k \rightarrow \infty} \sigma_{k}\left[\left(\frac{\left|e_{k+1}^{*}\right|}{1+\sigma_{k} \boldsymbol{H}_{k} \boldsymbol{P}_{k-1} \boldsymbol{H}_{k}^{T}}\right)^{2}-\triangle^{2}\right]=0$
(b) $\quad \limsup _{k \rightarrow \infty}\left(\frac{\left|e_{k+1}^{*}\right|^{2}}{\left(1+\sigma_{k} \boldsymbol{H}_{k} \boldsymbol{P}_{k-1} \boldsymbol{H}_{k}^{T}\right)^{2}}\right) \leq \triangle^{2}$

Proof. Define

$$
\gamma_{k} \triangleq \frac{1}{1+\sigma_{k} \boldsymbol{H}_{k} \boldsymbol{P}_{k-1} \boldsymbol{H}_{k}^{T}}
$$

Equation (42) can be obtained from (22) and (17), and

$$
\tilde{\boldsymbol{\beta}}_{k+1}=\tilde{\boldsymbol{\beta}}_{k}-\sigma_{k} \gamma_{k} \boldsymbol{P}_{k-1} \boldsymbol{H}_{k}^{T} e_{k+1}^{*}
$$


where $\tilde{\boldsymbol{\beta}}_{k+1}=\boldsymbol{\beta}^{*}-\hat{\boldsymbol{\beta}}_{k+1}$.

Equation (43) can be obtained from Assumption 4 and (42), and

$$
\begin{aligned}
\boldsymbol{H}_{k} \tilde{\boldsymbol{\beta}}_{k+1}+\triangle_{k} & =\boldsymbol{H}_{k} \tilde{\boldsymbol{\beta}}_{k+1}+\frac{y_{k+1}-y_{k}}{\triangle u_{k}}-\boldsymbol{H}_{k} \boldsymbol{\beta}^{*} \\
& =\boldsymbol{H}_{k} \tilde{\boldsymbol{\beta}}_{k}-\boldsymbol{H}_{k} \sigma_{k} \gamma_{k} \boldsymbol{P}_{k-1} \boldsymbol{H}_{k}^{T} e_{k+1}^{*}+\frac{y_{k+1}-y_{k}}{\triangle u_{k}}-\boldsymbol{H}_{k} \boldsymbol{\beta}^{*} \\
& =\frac{y_{k+1}-y_{k}}{\triangle u_{k}}-\boldsymbol{H}_{k} \hat{\boldsymbol{\beta}}_{k}-\boldsymbol{H}_{k} \sigma_{k} \gamma_{k} \boldsymbol{P}_{k-1} \boldsymbol{H}_{k}^{T} e_{k+1}^{*} \\
& =e_{k+1}^{*}-\frac{\boldsymbol{H}_{k} \sigma_{k} \boldsymbol{P}_{k-1} \boldsymbol{H}_{k}^{T} e_{k+1}^{*}}{1+\sigma_{k} \boldsymbol{H}_{k} \boldsymbol{P}_{k-1} \boldsymbol{H}_{k}^{T}} \\
& =\gamma_{k} e_{k+1}^{*}
\end{aligned}
$$

Introducing Lyapunov candidate as $V_{k+1}=\tilde{\boldsymbol{\beta}}_{k+1}^{T} \boldsymbol{P}_{k}^{-1} \tilde{\boldsymbol{\beta}}_{k+1}$, taking (20) into it, hence, we can obtain

$$
\begin{aligned}
V_{k+1} & =\tilde{\boldsymbol{\beta}}_{k+1}^{T} \boldsymbol{P}_{k}^{-1} \tilde{\boldsymbol{\beta}}_{k+1} \\
& =\tilde{\boldsymbol{\beta}}_{k+1}^{T} \boldsymbol{P}_{k-1}^{-1} \tilde{\boldsymbol{\beta}}_{k+1}+\sigma_{k} \tilde{\boldsymbol{\beta}}_{k+1}^{T} \boldsymbol{H}_{k}^{T} \boldsymbol{H}_{k} \tilde{\boldsymbol{\beta}}_{k+1} \\
& =\left[\tilde{\boldsymbol{\beta}}_{k}-\sigma_{k} \gamma_{k} \boldsymbol{P}_{k-1} \boldsymbol{H}_{k}^{T} e_{k+1}^{*}\right]^{T} \boldsymbol{P}_{k-1}^{-1}\left[\tilde{\boldsymbol{\beta}}_{k}-\sigma_{k} \gamma_{k} \boldsymbol{P}_{k-1} \boldsymbol{H}_{k}^{T} e_{k+1}^{*}\right]+\sigma_{k} \tilde{\boldsymbol{\beta}}_{k+1}^{T} \boldsymbol{H}_{k}^{T} \boldsymbol{H}_{k} \tilde{\boldsymbol{\beta}}_{k+1} \\
& =V_{k}-2 \sigma_{k} \gamma_{k} \boldsymbol{H}_{k} \tilde{\boldsymbol{\beta}}_{k} e_{k+1}^{*}+\sigma_{k}^{2} \gamma_{k}^{2}\left(e_{k+1}^{*}\right)^{2} \boldsymbol{H}_{k} \boldsymbol{P}_{k-1} \boldsymbol{H}_{k}^{T}+\sigma_{k}\left(\boldsymbol{H}_{k} \tilde{\boldsymbol{\beta}}_{k+1}\right)^{2}
\end{aligned}
$$

Substitute (43) in the above equation then

$$
\begin{aligned}
V_{k+1} & =V_{k}-2 \sigma_{k} \gamma_{k} \boldsymbol{H}_{k} \tilde{\boldsymbol{\beta}}_{k} e_{k+1}^{*}+\sigma_{k}^{2} \gamma_{k}^{2}\left(e_{k+1}^{*}\right)^{2} \boldsymbol{H}_{k} \boldsymbol{P}_{k-1} \boldsymbol{H}_{k}^{T}+\sigma_{k}\left(\boldsymbol{H}_{k} \tilde{\boldsymbol{\beta}}_{k+1}\right)^{2} \\
& =V_{k}-2 \sigma_{k} \boldsymbol{H}_{k} \tilde{\boldsymbol{\beta}}_{k}\left(\boldsymbol{H}_{k} \tilde{\boldsymbol{\beta}}_{k+1}+\triangle_{k}\right)+\sigma_{k}^{2} \boldsymbol{H}_{k} \boldsymbol{P}_{k-1} \boldsymbol{H}_{k}^{T}\left(\boldsymbol{H}_{k} \tilde{\boldsymbol{\beta}}_{k+1}+\triangle_{k}\right)^{2}+\sigma_{k}\left(\boldsymbol{H}_{k} \tilde{\boldsymbol{\beta}}_{k+1}\right)^{2} \\
& =V_{k}-2 \sigma_{k} \boldsymbol{H}_{k}\left(\tilde{\boldsymbol{\beta}}_{k+1}+\sigma_{k} \gamma_{k} \boldsymbol{P}_{k-1} \boldsymbol{H}_{k}^{T} e_{k+1}^{*}\right)\left(\boldsymbol{H}_{k} \tilde{\boldsymbol{\beta}}_{k+1}+\triangle_{k}\right)+\sigma_{k}^{2} \boldsymbol{H}_{k} \boldsymbol{P}_{k-1} \boldsymbol{H}_{k}^{T}\left(\boldsymbol{H}_{k} \tilde{\boldsymbol{\beta}}_{k+1}+\triangle_{k}\right)^{2} \\
& +\sigma_{k}\left(\boldsymbol{H}_{k} \tilde{\boldsymbol{\beta}}_{k+1}\right)^{2} \\
& =V_{k}-\sigma_{k}\left(\boldsymbol{H}_{k} \tilde{\boldsymbol{\beta}}_{k+1}\right)^{2}-2 \sigma_{k} \boldsymbol{H}_{k} \tilde{\boldsymbol{\beta}}_{k+1} \triangle_{k}-\sigma_{k}^{2} \boldsymbol{H}_{k} \boldsymbol{P}_{k-1} \boldsymbol{H}_{k}^{T}\left(\boldsymbol{H}_{k} \tilde{\boldsymbol{\beta}}_{k+1}+\triangle_{k}\right)^{2} \\
& \leq V_{k}-\sigma_{k}\left(\boldsymbol{H}_{k} \tilde{\boldsymbol{\beta}}_{k+1}\right)^{2}-2 \sigma_{k} \boldsymbol{H}_{k} \tilde{\boldsymbol{\beta}}_{k+1} \triangle_{k} \\
& =V_{k}+\sigma_{k} \triangle_{k}^{2}-\sigma_{k}\left(\boldsymbol{H}_{k} \tilde{\boldsymbol{\beta}}_{k+1}+\triangle_{k}\right)^{2}
\end{aligned}
$$

then

$$
\begin{aligned}
V_{k+1} & \leq V_{k}+\sigma_{k} \triangle_{k}^{2}-\sigma_{k}\left(\boldsymbol{H}_{k} \tilde{\boldsymbol{\beta}}_{k+1}+\triangle_{k}\right)^{2} \\
& =V_{k}+\sigma_{k} \triangle_{k}^{2}-\sigma_{k} \gamma_{k}^{2}\left(e_{k+1}^{*}\right)^{2} \\
& =V_{k}-\sigma_{k}\left(\gamma_{k}^{2}\left(e_{k+1}^{*}\right)^{2}-\triangle_{k}^{2}\right) \\
& \leq V_{k}-\sigma_{k}\left(\gamma_{k}^{2}\left(e_{k+1}^{*}\right)^{2}-\triangle^{2}\right) \\
V_{k+1} & -V_{k} \leq-\sigma_{k}\left(\gamma_{k}^{2}\left(e_{k+1}^{*}\right)^{2}-\triangle^{2}\right) \leq 0
\end{aligned}
$$

and $V_{k}$ is nonnegative.

Then

$$
\tilde{\boldsymbol{\beta}}_{k+1}^{T} \boldsymbol{P}_{k}^{-1} \tilde{\boldsymbol{\beta}}_{k+1} \leq \tilde{\boldsymbol{\beta}}_{k}^{T} \boldsymbol{P}_{k-1}^{-1} \tilde{\boldsymbol{\beta}}_{k} \leq \tilde{\boldsymbol{\beta}}_{1}^{T} \boldsymbol{P}_{0}^{-1} \tilde{\boldsymbol{\beta}}_{1} .
$$

The following inequalities can be obtained from the matrix inversion lemma,

$$
\lambda_{\min }\left(\boldsymbol{P}_{k}^{-1}\right) \geq \lambda_{\min }\left(\boldsymbol{P}_{k-1}^{-1}\right) \geq \lambda_{\min }\left(\boldsymbol{P}_{0}^{-1}\right),
$$


and this implies

$$
\begin{aligned}
\lambda_{\min }\left(\boldsymbol{P}_{0}^{-1}\right)\left\|\tilde{\boldsymbol{\beta}}_{k+1}\right\|^{2} & \leq \lambda_{\min }\left(\boldsymbol{P}_{k}^{-1}\right)\left\|\tilde{\boldsymbol{\beta}}_{k+1}\right\|^{2} \\
& \leq \tilde{\boldsymbol{\beta}}_{k+1}^{T} \boldsymbol{P}_{k}^{-1} \tilde{\boldsymbol{\beta}}_{k+1} \\
& \leq \tilde{\boldsymbol{\beta}}_{1}^{T} \boldsymbol{P}_{0}^{-1} \tilde{\boldsymbol{\beta}}_{1} \\
& \leq \lambda_{\max }\left(\boldsymbol{P}_{0}^{-1}\right)\left\|\tilde{\boldsymbol{\beta}}_{1}\right\| .
\end{aligned}
$$

This establishes part (1).

Summing both sides of (47) from 1 to $N$ with $V_{k}$ being nonnegative, we have

$$
V_{k+1} \leq V_{1}-\lim _{N \rightarrow \infty} \sum_{k=1}^{N} \sigma_{k}\left(\gamma_{k}^{2}\left(e_{k+1}^{*}\right)^{2}-\triangle^{2}\right),
$$

and we immediately get

$$
\lim _{N \rightarrow \infty} \sum_{k=1}^{N} \sigma_{k}\left[\frac{\left(e_{k+1}^{*}\right)^{2}}{\left(1+\sigma_{k} \boldsymbol{H}_{k} \boldsymbol{P}_{k-1} \boldsymbol{H}_{k}^{T}\right)^{2}}-\triangle^{2}\right] \leq \infty
$$

(2) holds, then

$$
\lim _{k \rightarrow \infty} \sigma_{k}\left[\frac{\left(e_{k+1}^{*}\right)^{2}}{\left(1+\sigma_{k} \boldsymbol{H}_{k} \boldsymbol{P}_{k-1} \boldsymbol{H}_{k}^{T}\right)^{2}}-\triangle^{2}\right]=0
$$

(a) and (b) hold.

We also immediately have

$$
\limsup _{k \rightarrow \infty}\left[\frac{e_{k+1}^{*}}{\left(1+\sigma_{k} \boldsymbol{H}_{k} \boldsymbol{P}_{k-1} \boldsymbol{H}_{k}^{T}\right)}\right]^{2} \leq \triangle^{2}
$$

Because the activation function $G\left(w_{i}, b_{i}, x_{j}\right)$ is bounded, $e_{k+1}^{*}$ is bounded. Because $\triangle u_{k}=\left|\frac{y_{k+1}^{*}-y_{k}}{\boldsymbol{H}_{k} \hat{\beta}_{k}}\right| \leq M, e_{k+1}=y_{k+1}-y_{k+1}^{*}$ is bounded.

\section{Analysis of Experimental Results}

In this paper, EMREOS-ELM is investigated, and EMREOS-ELM is adopted for estimating the value $\psi_{k}$; EMREOS-ELM is an online learning algorithm and can train a single hidden layer neural network; besides it can adjust the network structure. In order to further show the effectiveness of the MFAC method based on EMREOS-ELM, the MFAC method based on another online learning algorithm is compared, and the online learning algorithm adjusts network structure using an incremental form. In order to describe this online learning algorithm easily, in this paper, this online learning algorithm is named as IREOS-ELM. The MFAC method based on IREOS-ELM also has three phases, parameter initialization, parameter learning and the adjustment of network structure. During the adjustment of network structure, all input weights and all hidden biases are reinitialized, and the network is retrained by using the $k-1$ th training data. The MFAC method based on EMREOS-ELM is different from the MFAC method based on IREOS-ELM, it only initializes the input weight of the increased hidden node and the hidden bias of the increased hidden node, and it updates the output weights using the pseudoinverse of a partitioned matrix.

In this part, a simulation experiment is did, besides the MFAC algorithm based on EMREOS-ELM is compared with the MFAC algorithm based on RLS ([33] Equation 5.49), the MFAC method based on IREOS-ELM and RBF algorithm ([34] Equation 10.22). 
In this numerical simulation experiment, the following single-input single-output unknown discrete-time nonlinear system is considered, and

$$
y_{k+1}=\frac{y_{k}}{1+y_{k}^{2}}+u_{k}^{3}
$$

The discrete-time nonlinear system (55) comes from [35].

The reference signal is

$$
y_{k+1}^{*}=4+2 \sin \left(\pi T_{s} k\right)+1.5 \cos \left(\pi T_{s} k\right)
$$

where $T_{s}$ is the sampling time, and $T_{s}=0.01$.

The parameters of MFAC algorithm based on RLS are $\rho_{k}=1, \lambda_{1}=0.01$, and $a_{0}=0.95$. The activation function of EMREOS-ELM is sigmoid, $\lambda=0.1$, and $\triangle=0.002$. Figures 2-6 show the main results of this numerical simulation experiment. Figure 2 plots the tracking curves, and in Figure 3, the curve denotes the tracking error change trend. Figure 4 shows the change trends of $E_{k}$ and $L_{k}$. Figure 4 indicates that the final value of $L_{k}$ is 9 , when $L_{k}=9$, the tracking error satisfies $E_{k} \leq E_{m}$, and the result of Figure 4 shows that EMEROS-ELM is effective in obtaining a compact network structure. Figure 5 indicates the change curve of $\hat{\psi}_{k}, \hat{\psi}_{k}=\boldsymbol{H}_{k} \hat{\boldsymbol{\beta}}_{k}$, and $\hat{\psi}_{k}$ is the estimated value of $\psi_{k}$. In Section 4.4, the stability analysis of the MFAC algorithm based on EMREOS-ELM for unknown discrete-time nonlinear was stated, and we get the inequality (47). In order to verify the inequality (47), a new variable as

$$
\Delta v_{k} \triangleq-\sigma_{k}\left(\gamma_{k}^{2}\left(e_{k+1}^{*}\right)^{2}-\triangle^{2}\right) .
$$

Figure 6 shows the change curve of of $\Delta v_{k}$, and it indicates that $\Delta v_{k} \leq 0$.

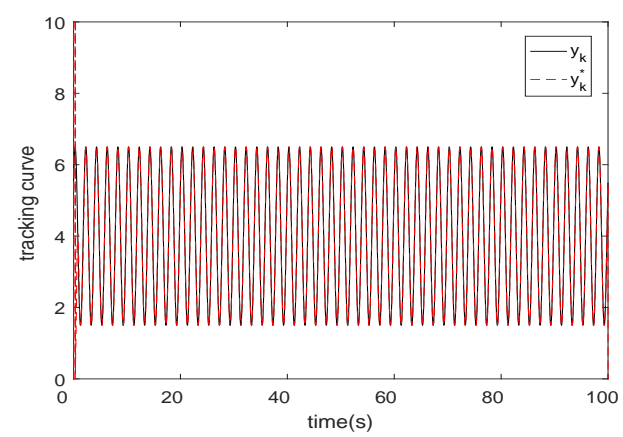

Figure 2. The tracking curves of simulation experiment. 


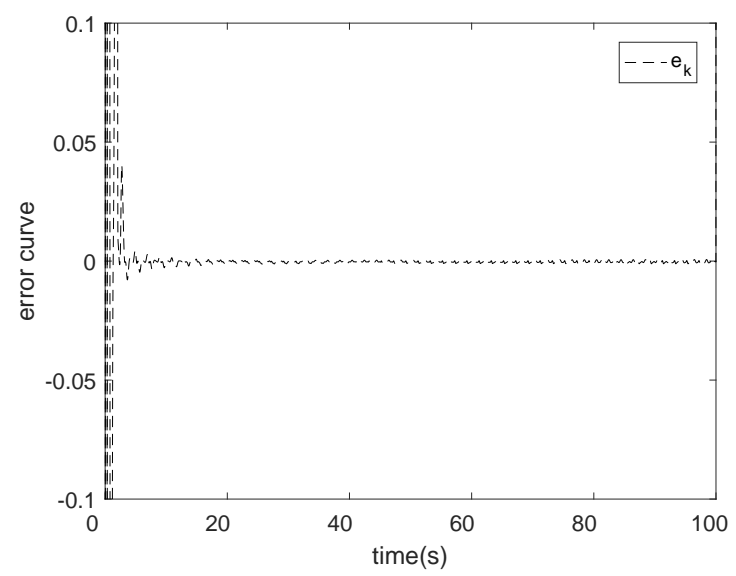

Figure 3. The changing trend of $e_{k}$.
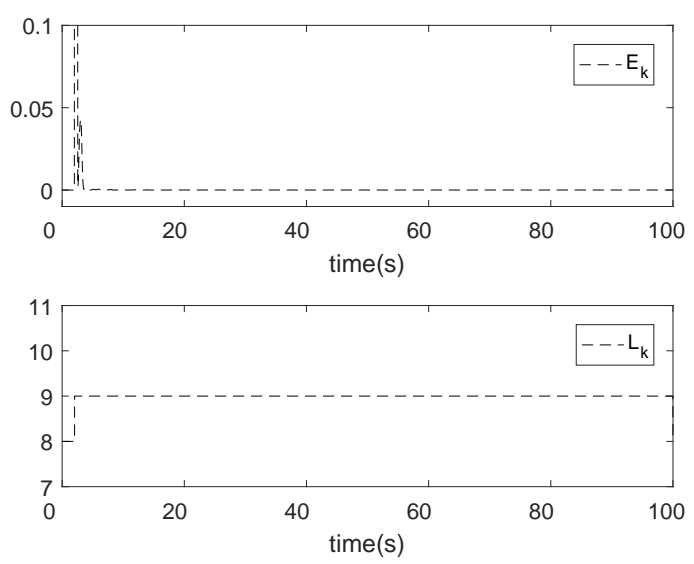

Figure 4. The changing trends of $E_{k}$ and $L_{k}$.

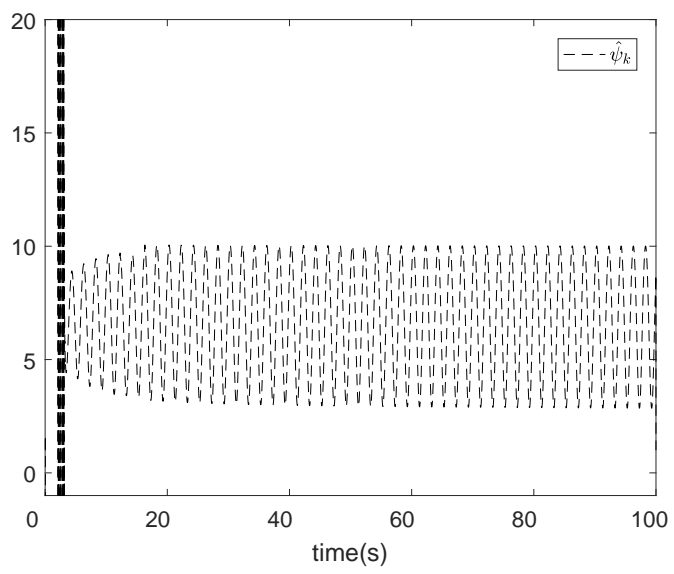

Figure 5. The changing trend of $\hat{\psi}_{k}$. 


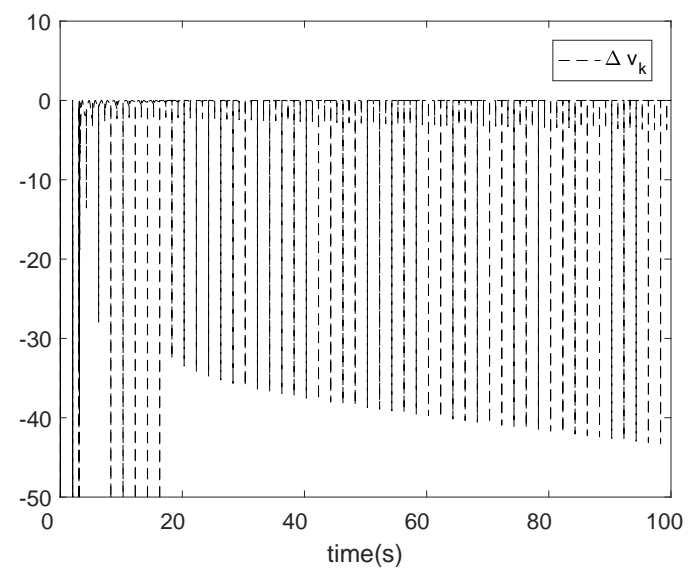

Figure 6. The changing trend of $\Delta v_{k}$.

Besides the error change curves of $e_{k}^{(1)}, e_{k}^{(2)}, e_{k}^{(3)}$ and $e_{k}^{(4)}$ are plotted in Figures 7-10, respectively. Here $e_{k}^{(1)}$ indicates the error change curve of the REOS-ELM which has a fixed network structure, and the number of hidden nodes is $8 ; e_{k}^{(2)}$ indicates the error change curve of the REOS-ELM which has a fixed network structure, and the number of hidden nodes is $20 ; e_{k}^{(3)}$ is the error change curve of the MFAC algorithm based on RLS; and $e_{k}^{(4)}$ indicates the error change curve of RBF which has a fixed network structure, and the number of hidden nodes is 9 . Figures 7-10 indicate that the tracking effect of EMREOS-ELM is better than four other algorithms.

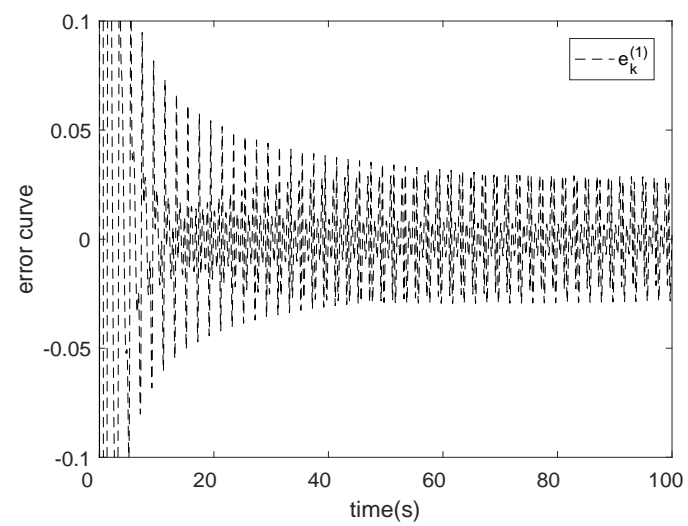

Figure 7. The changing trend of $e_{k}^{(1)}$.

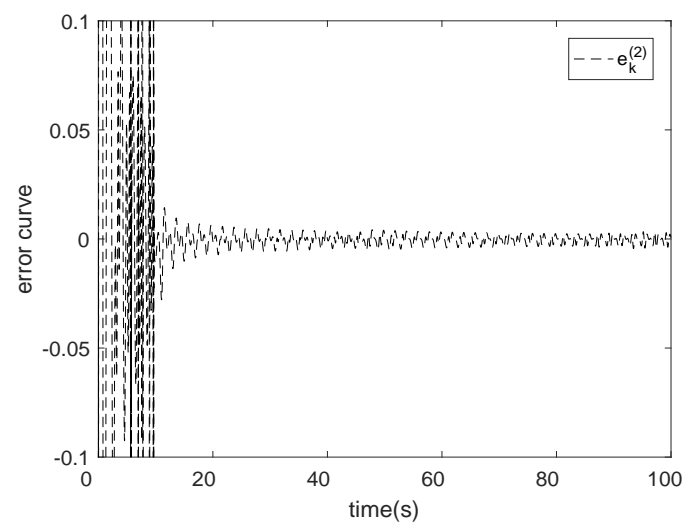

Figure 8. The changing trend of $e_{k}^{(2)}$. 


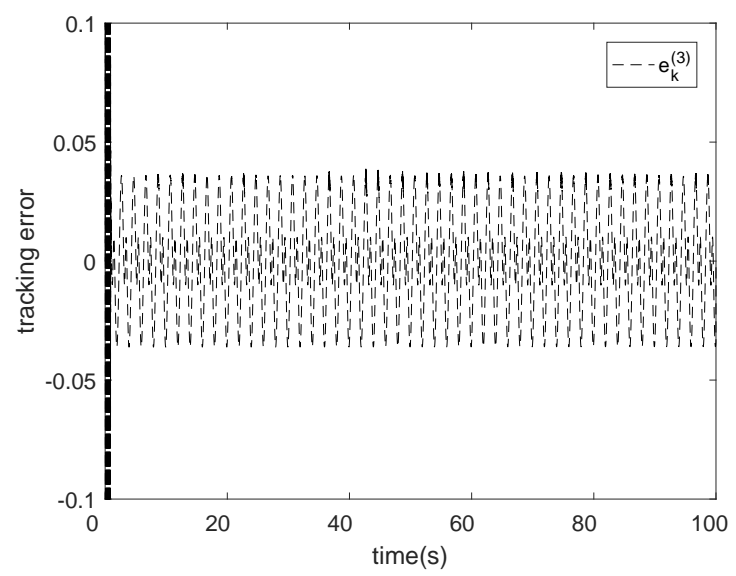

Figure 9. The changing trend of $e_{k}^{(3)}$.

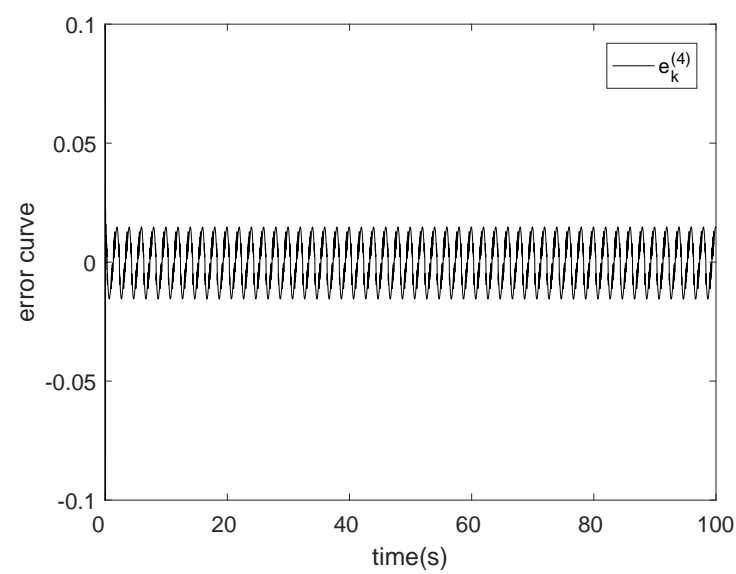

Figure 10. The changing trend of $e_{k}^{(4)}$.

In order to further show the effectiveness of algorithms, the integral square error (ISE) index of predicted output is introduced, and

$$
e_{\mathrm{ISE}}=\sum_{l=5000}^{10000}\left(y_{l}^{*}-y_{l}\right)^{2} .
$$

Twenty groups of simulation experiments were done, and the ISE of those six control algorithms are shown in Table 1. The final hidden node numbers of simulation experiments are showed in Table 2, and Table 2 shows that the MFAC method based on EMREOS-ELM is effective in adjusting network structure. The average values of ISE indicate that compared with five other control algorithms, the proposed algorithm has an improvement in the performance of control systems. 
Table 1. The simulation result $e_{\mathrm{ISE}}$.

\begin{tabular}{|c|c|c|c|c|c|c|}
\hline & $\begin{array}{c}\text { The MFAC } \\
\text { Algorithm } \\
\text { Based on } \\
\text { REOS-ELM }(\mathrm{L}=8)\end{array}$ & $\begin{array}{c}\text { The MFAC } \\
\text { Algorithm } \\
\text { Based on } \\
\text { REOS-ELM }(\mathrm{L}=20)\end{array}$ & $\begin{array}{l}\text { The MFAC } \\
\text { Algorithm } \\
\text { Based on } \\
\text { EMREOS-ELM }\end{array}$ & $\begin{array}{c}\text { RBFNN } \\
(\mathrm{L}=9)\end{array}$ & $\begin{array}{l}\text { The MFAC } \\
\text { Algorithm } \\
\text { Based } \\
\text { on RLS }\end{array}$ & $\begin{array}{c}\text { The MFAC } \\
\text { Algorithm } \\
\text { Based on } \\
\text { IREOS-ELM }\end{array}$ \\
\hline 1 & 0.150672 & 0.002515 & 0.001562 & 0.550053 & 4.449964 & 0.012889 \\
\hline 2 & 0.019459 & 0.040914 & 0.001705 & 0.502426 & 2.534129 & 0.433985 \\
\hline 3 & 0.703282 & 0.014989 & 0.005451 & 0.564361 & 2.274406 & 0.016331 \\
\hline 4 & 0.172610 & 0.150750 & 0.027222 & 0.504968 & 1.909120 & 0.017123 \\
\hline 5 & 0.083149 & 0.244061 & 0.004806 & 0.402855 & 1.824217 & 0.007621 \\
\hline 6 & 0.696841 & 0.009112 & 0.006475 & 0.411857 & 2.548398 & 0.004997 \\
\hline 7 & 1.568039 & 0.051220 & 0.014730 & 0.591908 & 2.222298 & 0.182836 \\
\hline 8 & 0.322919 & 0.024096 & 0.00295 & 0.584721 & 1.839572 & 0.009986 \\
\hline 9 & 0.051606 & 0.576331 & 0.00295 & 0.524870 & 1.799829 & 0.048388 \\
\hline 10 & 0.158293 & 0.678617 & 0.003559 & 0.696158 & 2.231448 & 0.001695 \\
\hline 11 & 0.055251 & 0.004448 & 0.014255 & 0.551650 & 2.409947 & 0.015570 \\
\hline 12 & 0.008733 & 0.004131 & 0.019071 & 0.493070 & 1.913370 & 0.046355 \\
\hline 13 & 0.376944 & 0.008184 & 0.004649 & 0.553147 & 2.946602 & 0.012967 \\
\hline 14 & 0.297452 & 0.01459 & 0.002863 & 0.633631 & 1.906357 & 0.054175 \\
\hline 15 & 0.132094 & 0.329650 & 0.002116 & 0.730532 & 2.456959 & 0.016178 \\
\hline 16 & 0.099835 & 0.194384 & 0.036917 & 0.779908 & 3.446312 & 0.017376 \\
\hline 17 & 0.222907 & 0.002091 & 0.011495 & 0.431327 & 1.952456 & 0.049845 \\
\hline 18 & 0.021288 & 0.002629 & 0.005704 & 0.561705 & 2.045576 & 0.049984 \\
\hline 19 & 0.481443 & 0.004517 & 0.025093 & 0.582250 & 2.380352 & 0.007150 \\
\hline 20 & 0.018625 & 0.212689 & 0.001724 & 0.664968 & 2.012102 & 0.016439 \\
\hline average value & 0.282072 & 0.128496 & 0.009729 & 0.565818 & 2.354829 & 0.051094 \\
\hline
\end{tabular}

Table 2. The final number of hidden nodes.

\begin{tabular}{|c|c|c|c|c|c|c|c|c|c|c|c|c|c|c|c|c|c|c|c|c|}
\hline & 1 & 2 & 3 & 4 & 5 & 6 & 7 & 8 & 9 & 10 & 11 & 12 & 13 & 14 & 15 & 16 & 17 & 18 & 19 & 20 \\
\hline $\begin{array}{l}\text { The MFAC algorithm } \\
\text { based on EMREOS-ELM }\end{array}$ & 9 & 9 & 10 & 11 & 10 & 14 & 9 & 9 & 10 & 10 & 10 & 10 & 9 & 11 & 10 & 11 & 11 & 11 & 10 & 9 \\
\hline $\begin{array}{l}\text { The MFAC algorithm } \\
\text { based on IREOS-ELM }\end{array}$ & 10 & 11 & 10 & 9 & 12 & 11 & 11 & 11 & 10 & 10 & 12 & 10 & 9 & 11 & 10 & 14 & 11 & 9 & 9 & 10 \\
\hline
\end{tabular}

\section{Conclusions}

In order to analyse the stability of the MFAC algorithm based on REOS-ELM for unknown discrete-time nonlinear systems, an updating formula, which contains dead-zone characteristics, is introduced, and EMREOS-EM is investigated for the purpose of getting a more compact network structure and improving the performances of control systems. The proposed MFAC method based on EMREOS-ELM is compared with five other algorithms, and the simulation results show that the proposed algorithm has an improvement in the performance of control systems.

In this paper, the MFAC method based on EMREOS-ELM is introduced for single-input single-output unknown discrete-time nonlinear systems. In the future, we plan to do some work for multiple-input, multiple-output unknown discrete-time nonlinear systems based on the proposed algorithm, and in order to improve the performance of robot control systems, we will also apply this algorithm to robot control systems which are complex nonlinear systems.

Author Contributions: Conceptualization and Methodology, X.Z. and H.M.; Writing-draft version, X.Z.; Writing-review editing, X.Z. and H.M.

Funding: This work is partially supported by Ministry of Science and Technology of the People's Republic of China under Grant No. 2017YFF0205306. This work is also partially supported by National natural Science Foundation of China under Grant No. 91648117.

Conflicts of Interest: The authors declare no conflict of interest. 


\section{Abbreviations}

The following abbreviations are used in this manuscript:

$\begin{array}{ll}\text { MFAC } & \text { model free adaptive control } \\ \text { I/O } & \text { input/output } \\ \text { DDC } & \text { data-driven control } \\ \text { PID } & \text { proportional-integral derivative } \\ \text { UC } & \text { unfalsified control } \\ \text { RBFNN } & \text { radial basis function neural network } \\ \text { EMREOS-ELM } & \text { error minimized regularized online sequential extreme learning machine } \\ \text { ELM } & \text { extreme learning machine } \\ \text { OS-ELM } & \text { online sequential extreme learning machine } \\ \text { REOS-ELM } & \text { regularized online sequential extreme learning machine } \\ \text { EMOS-ELM } & \text { error minimized online sequential extreme learning machine }\end{array}$

\section{References}

1. Hou, Z.S.; Xu, J.X. On data-driven control theory: The state of the art and perspective. Acta Autom. Sin. 2009, 35, 650-667. [CrossRef]

2. Caponetto, R.; Dongola, G.; Fortuna, L.; Gallo, A. New results on the synthesis of FO-PID controllers. Commun. Nonlinear Sci. 2010, 15, 997-1007. [CrossRef]

3. Roman, R.C.; Precup, R.E.; David, R.C. Second order intelligent proportional-integral fuzzy control of twin rotor aerodynamic systems. Procedia Comput. Sci. 2018, 139, 372-380. [CrossRef]

4. Sanchez-Pena, R.S.; Colmegna, P.; Bianchi, F. Unfalsified control based on the controller parameterisation. Int. J. Syst. Sci. 2015, 46, 2820-2831. [CrossRef]

5. Safonov, M.G.; Tsao, T.C. The unfalsfied control concept and learning. IEEE Trans. Autom. Control 1997, 42, 2819-2824. [CrossRef]

6. Hou, Z.S.; Huang, W.H. The model-free learning adaptive control of a class of SISO nonlinear systems. In Proceedings of the 1997 American Control Conference, Albuquerque, NM, USA, 6 June 1997; Volume 1, pp. 343-344.

7. Hou, Z.S.; Jin, S.T. A novel data-driven control approach for a class of discrete-time nonlinear systems. IEEE Trans. Control Syst. Technol. 2011, 19, 1549-1558. [CrossRef]

8. Tutsoy, O.; Barkana, D.E.; Tugal, H. Design of a completely model free adaptive control in the presence of parametric, non-parametric uncertainties and random control signal delay. ISA Trans. 2018, 76, 67-77. [CrossRef]

9. Safaei, A.; Mahyuddin, M.N. Adaptive model-free control based on an ultra-local model with model-free parameter estimations for a generic SISO system. IEEE Access 2018, 6, 4266-4275. [CrossRef]

10. Hung-Yi, C.; Jin-Wei, L. Model-free adaptive sensing and control for a piezoelectrically actuated system. Sensors 2010, 10, 10545-10559.

11. Xia, Y.; Dai, Y.; Yan, W.; Xu, D.; Yang, C. Adaptive-observer-based data driven voltage control in islanded-mode of distributed energy resource systems. Energies 2018, 11, 3299. [CrossRef]

12. Hou, Z.S.; Xiong, S.S. On model free adaptive control and its stability analysis. IEEE Trans. Autom. Control 2019, in press, doi:10.1109/TAC.2019.2894586 . [CrossRef]

13. He, W.; Meng, T.T.; He, X.Y.; Samge, S.Z. Unified iterative learning control for flexible structures with input constraints. Automatica 2018, 96, 326-336. [CrossRef]

14. Xuan, Y.; Ruan, X. Reinforced gradient-type iterative learning control for discrete linear time-invariant systems with parameters uncertainties and external noises. IMA J. Math. Control Inf. 2016, 34, 1117-1133.

15. Miao, Y.; Wei, Z.; Liu, B.B. On iterative learning control for MIMO nonlinear systems in the presence of time-iteration-varying parameters. Nonlinear Dynam. 2017, 89, 2561-2571.

16. Hjalmarsson, H. Iterative feedback tuning-An overview. Int. J. Adapt. Control Signal Process. 2010, 16, 373-95. [CrossRef]

17. Heertjes, M.F.; Velden, B.V.D.; Oomen, T. Constrained iterative feedback tuning for robust control of a wafer stage system. IEEE Trans. Control Syst. Technol. 2016, 24, 56-66. [CrossRef] 
18. RiosPatron, E.; Braatz, R. On the identification and control of dynamical systems using neural networks. IEEE Trans. Neural Netw. 1997, 8, 452.

19. Ren, X.M.; Lewis, F.L.; Zhang, J.L. Neural network compensation control for mechanical systems with disturbances. Automatic 2009, 45, 1221-1226. [CrossRef]

20. Wang, F.; Chen, B.; Lin, C.; Zhang, J.; Meng, X. Adaptive neural network finite-time output feedback control of quantized nonlinear systems. IEEE Trans. Cybern. 2018, 48, 1839-1848. [CrossRef]

21. Sun, C.Y.; He, W.; Ge, W.L.; Chang, C. Adaptive neural network control of biped robots. IEEE Trans. Syst. Man Cybern. Syst. 2017, 47, 315-326. [CrossRef]

22. Wang, M.S.; Tsai, T.M. Sliding mode and neural network control of sensorless PMSM controlled system for power consumption and performance improvement. Energies 2017, 10, 1780. [CrossRef]

23. Faria, J.; Pombo, J.; Calado, M.D.R.; Mariano, S. Power management control strategy based on artificial neural networks for standalone PV applications with a hybrid energy storage system. Energies 2019, 12, 902. [CrossRef]

24. Hou, Z.S.; Liu, S.D.; Tian, T.T. Lazy-learning-based data-driven model-free adaptive predictive control for a class of discrete-time nonlinear systems. IEEE Trans. Neural Netw. Learn. Syst. 2016, 28, 1-15. [CrossRef] [PubMed]

25. Zhu, Y.M.; Hou, Z.S. Data-driven MFAC for a class of discrete-time nonlinear systems with RBFNN. IEEE Trans. Neural Netw. Learn. Syst. 2014, 25, 1013-1020. [PubMed]

26. Huang, G.B.; Zhu, Q.Y.; Siew, C.K. Extreme learning machine: Theory and applications. Neurocomputing 2006, 70, 489-501. [CrossRef]

27. Liang, N.Y.; Huang, G.B.; Saratchandran, P.; Sundararajan, N. A fast and accurate online sequential learning algorithm for feedforward networks. IEEE Trans. Neural Netw. 2006, 17, 1411-1423. [CrossRef] [PubMed]

28. Jia, C.; Li, X.L.; Wang, K.; Ding, D.W. Adaptive control of nonlinear system using online error minimum neural networks. ISA Trans. 2016, 65, 125-132. [CrossRef] [PubMed]

29. Li, X.L.; Jia, C.; Liu, D.X.; Ding, D.W. Adaptive control of nonlinear discrete-time systems by using OS-ELM neural networks. Abstr. Appl. Anal. 2014, 2014, 1-11. [CrossRef]

30. Huynh, H.T.; Won, Y. Regularized online sequential learning algorithm for single-hidden layer feedforward neural networks. Pattern Recognit. Lett. 2011, 32, 1930-1935. [CrossRef]

31. Gao, X.H.; Wong, K.I.; Wong, P.K.; Chi, M.V. Adaptive control of rapidly time-varying discrete-time system using initial-training-free online extreme learning machine. Neurocomputing 2016, 194, 117-125. [CrossRef]

32. Huang, G.B.; Chen, L.; Siew, C.K. Universal approximation using incremental constructive feedforward networks with random hidden nodes. IEEE Trans. Neural Netw. 2006, 17, 879-892. [CrossRef] [PubMed]

33. Hong, Z.S. NonParametric Model and Adaptive Control Theory; Science Press: Beijing, China, 1999.

34. Kun, L.J. RBF Neural Network Adaptive Control Matlab Simulation; Tsinghua University Pre: Beijing, China, 2014.

35. Narendra, K.S. Identification and control for dynamic systems using neural networks using neural network. IEEE Trans. Inform. Theory 1990, 1, 4-27.

(c) 2019 by the authors. Licensee MDPI, Basel, Switzerland. This article is an open access article distributed under the terms and conditions of the Creative Commons Attribution (CC BY) license (http:/ / creativecommons.org/licenses/by/4.0/). 